\title{
Antimicrobial Properties of Actinomycetes Isolated from Menengai Crater in Kenya
}

\author{
Paul Njenga Waithaka1 ${ }^{*}$, Francis B. Mwaura1, John M. Wagacha1, Eliud M. Gathuru ${ }^{2}$, \\ Benson M. Githaiga ${ }^{2}$
}

${ }^{1}$ School of Biological Sciences, University of Nairobi, Nairobi, Kenya

${ }^{2}$ Department of Biological Sciences, Egerton University, Njoro, Kenya

Email: ^waithakanj@gmail.com, waithakanj@yahoo.com

How to cite this paper: Waithaka, P.N., Mwaura, F.B., Wagacha, J.M., Gathuru, E.M. and Githaiga, B.M. (2017) Antimicrobial Properties of Actinomycetes Isolated from Menengai Crater in Kenya. CellBio, 6, 13-26.

https://doi.org/10.4236/cellbio.2017.62002

Received: April 11, 2017

Accepted: June 27, 2017

Published: June 30, 2017

Copyright $\odot 2017$ by authors and Scientific Research Publishing Inc. This work is licensed under the Creative Commons Attribution International License (CC BY 4.0).

http://creativecommons.org/licenses/by/4.0/

\begin{abstract}
A study was carried out to isolate and screen actinomycetes for antimicrobials from Menengai Crater in Kenya. The actinomycetes were isolated using starch casein agar, Luria Bertani agar and starch nitrate agar. Primary screening for antagonism was carried out using perpendicular method while secondary screening was done using agar disk technique. Extraction of the antimicrobials was carried out using ethyl acetate. Sensitivity testing of the crude extracts against Staphylococcus aureus, Bacillus subtilis, Escherichia faecalis, Escherichia coli, Klebsiella pneumoniae, Salmonella typhi, Xanthomonas campestris, Erwinia carotovora, Candida albicans, Alternaria alternate and Fusarium oxysporum was carried out using agar well technique. Biochemical tests and carbon source requirements were used in characterization of the selected antimicrobial producers. M1 was the best agar medium for isolation of actinomycetes. The number of actinomycetes from regions $\mathrm{A}, \mathrm{B}, \mathrm{C}$, and $\mathrm{D}$ in the crater varied significantly $(\mathrm{F}=27.50 P=0.000)$. Out of the 156 actinomycetes isolates, 20 isolates were positive for both primary and secondary screening for antimicrobials. There was no significant difference in the zones of inhibition in primary screening of the actinomycetes for antagonistic properties against the test pathogens $(\mathrm{F}=1.6957 P=0.0838)$. The zones of inhibition after secondary screening varied significantly $(\mathrm{F}=2.4473 P=0.0089)$. Likewise, there was a significant difference $(\mathrm{F}=6.6046 P=0.001338)$ in the zones of inhibition after exposing the pathogens to ethyl extracts of the selected antagonistic actinomycetes. There is need to purify and characterize the antimicrobials obtained from the present study.
\end{abstract}

\section{Keywords}

Actinomycetes, Antimicrobials, Crater, Menengai, Metabolites, Sensitivity 


\section{Introduction}

Naturally occurring antimicrobials are needed in the world today to cub the rising antimicrobial resistance among pathogenic micro-organisms [1]. Soils offer a favourable habitat for the growth of actinomycetes which are good producers of antimicrobials. The need for identifying undiscovered actinomycetes, which increases the likelihood of discovering new drugs is on the increase [2]. New antibiotics produced by such actinomycetes will help in solving challenges of drug resistance and in treating life-threatening diseases such as cancer [3].

Production of secondary metabolites by actinomycetes may offer solutions to these problems [4]. Currently, over 5000 antibiotics have been screened from Gram positive, Gram negative bacteria as well as fungi. However, only 100 of these antibiotics have been developed to the level of treating human, animal and plant diseases [5]. This has been attributed to toxicity of majority of the antibiotics [6].

Actinomycetes are said to be morphologically, physiologically and ecologically diverse organisms [7]. They are of great significance to the pharmaceutical world due to their ability to produce secondary metabolites of medical importance [8]. Most of their metabolites have been shown to have antibacterial (streptomycin, tetracycline, chloramphenicol), antifungal (nystatin), antiviral (tunicamycin), antiparasitic (avermectin) properties [9]. Indeed, most of the antimicrobials used today in remedying diseases caused by pathogens have been developed from actinomycetes.

Among the genera of actinomycetes, Streptomyces occupy the biggest ecological niche [10] and they produce the vast majority of antimicrobials known today. Nevertheless, the search for rare actinomycetes which can produce new antibiotics is still a fertile approach to the problem [11]. Besides increased chances of discovering antibiotics by searching for rare actinomycetes, exploring hostile environments such hot deserts, deep seas, saline environments and volcanic areas is another option [12]. This study was therefore carried out to isolate and screen actinomycetes for antimicrobials from the environmentally harsh Menengai Crater in Kenya.

\section{Materials and Methods}

\subsection{Description of the Study Area}

Soils were sampled from Menengai crater located in Rongai and Nakuru North sub-counties in Nakuru County, Kenya. The crater is located at an elevation of $2278 \mathrm{~m}$ above sea level. Currently, the crater is considered dormant but there are underground geothermal activities which raise the temperatures of the region to a maximum of $82^{\circ} \mathrm{C}$. After volcanic eruptions estimated to have occurred 2000 years ago, walls that were formed on its sides collapsed leading to formation of a large hole at the centre referred to as a caldera [13]. 


\subsection{Soil Sampling}

Based on land terrain and soil characteristics, the study area was divided into regions A, B, C, D and eight sampling points were randomly identified from each of the regions. Geothermal vents occurred in regions $A$ and $D$ and were referred to as vents A and vents D, respectively. Soil samples were separately collected from the top $5 \mathrm{~cm}$ using a sterile trowel and placed in zip lock bags. The samples were transported to the Department of Biological Sciences laboratory, Egerton University. The soil samples were air dried on the laboratory benches for one week to kill some of the vegetative microorganisms. Heat treatment of the samples was carried out by separately placing the samples in $250 \mathrm{ml}$ Erlenmeyer flask and holding them in a water bath at $50^{\circ} \mathrm{C}$ for $1 \mathrm{~h}$ to further reduce the number of vegetative types of other bacterial flora.

\subsection{Preparation of the Culture Media}

Actinomycetes were cultured on starch casein agar (SCA) (starch $10 \mathrm{~g}, \mathrm{~K}_{2} \mathrm{HPO}_{4} 2$ g, $\mathrm{KNO}_{3} 2 \mathrm{~g}$, casein $0.3 \mathrm{~g}, \mathrm{MgSO}_{4} \cdot 7 \mathrm{H}_{2} \mathrm{O} 0.05 \mathrm{~g}, \mathrm{CaCO}_{3} 0.02 \mathrm{~g}, \mathrm{FeSO}_{4} \cdot 7 \mathrm{H}_{2} \mathrm{O} 0.01 \mathrm{~g}$, agar $15 \mathrm{~g}$, filtered sea water $1000 \mathrm{ml}$ and $\mathrm{pH} 7.0 \pm 0.1$ ); Luria Bertani (M1) medium (starch 10 g, Peptone 2.0 g, Yeast Extract 4.0 g, Agar 18.0 g, distilled water $1000 \mathrm{ml}, \mathrm{pH} ; 7.0 \pm 0.1$ ) and starch nitrate agar (soluble starch $20.0 \mathrm{~g}, \mathrm{~K}_{2} \mathrm{HPO}_{4} 1.0$ g, $\mathrm{KNO}_{3} 2.0 \mathrm{~g}, \mathrm{MgSO}_{4} 0.5 \mathrm{G}, \mathrm{CaCO}_{3} 3.0 \mathrm{~g}, \mathrm{NaCl} 100 \mathrm{~g}, \mathrm{FeSO}_{4} 0.1 \mathrm{~g}, \mathrm{MnCl}_{2} 0.1 \mathrm{~g}$, $\mathrm{ZnSO}_{4} 0.1 \mathrm{~g}$, Distilled water $100 \mathrm{ml}, \mathrm{pH} 7.0 \pm 0.1$ ). The media were dissolved in distilled water as guided by the manufacturers prior to autoclaving at $121^{\circ} \mathrm{C}$ for $15 \mathrm{~min}$. The media were supplemented with $25 \mu \mathrm{g} \cdot \mathrm{ml}^{-1}$ nystatin do suppress growth of fungi and $10 \mu \mathrm{g} \cdot \mathrm{ml}^{-1}$ nalidixic acid to minimize growth of Gram negative and some Gram-positive bacteria.

\subsection{Isolation of Actinomycetes}

Separately $1 \mathrm{~g}$ of soil sample was added to $9 \mathrm{ml}$ of distilled water in a test tube. The test tubes were shaken in an orbital shaker rotating at $200 \mathrm{rpm}$ for $10 \mathrm{~min}$ to release actinomycetes that were strongly attached to the soil particles. Aseptically, serial dilution was carried out up to $10^{-6}$. Following this, $0.1 \mathrm{ml}$ of each sample was separately plated in the three-isolation media using spread plate technique. The plates were incubated at $30^{\circ} \mathrm{C}$ for up to one month. The growing colonies were identified as actinomycetes using cultural characteristics such as colonies that were tough, leathery, and partially submerged into the agar. Colonies having these characteristics were sub-cultured onto yeast extract malt extract agar medium and incubated at $30^{\circ} \mathrm{C}$ for up to one month. Sub-culturing was carried out until pure cultures were obtained. The pure cultures were preserved in slants and glycerol after coding using letters PAN followed by a number.

\section{Biochemical Characterization of Actinomycetes}

\subsection{Gram's Staining}

Crystal violet, gram's iodine, 95\% ethyl alcohol and safranin were used in Gram 
staining. Briefly, the isolated actinomycetes were separately placed on glass slides using a wire loop. Aseptically, crystal violet was added on the slides and allowed to stand for $1 \mathrm{~min}$. The excess stain was drained off using tap water. Following this, Gram's iodine was added and the preparation allowed to stand for another 1 min. The excess Gram's iodine was removed using running water. Ethyl alcohol was added dropwise followed by washing with running water. A counter stain safranin was added and allowed to stand for $45 \mathrm{~s}$ before observation of the culture under the microscope [14].

\subsection{Use of API Strips}

Biochemical characterization of the isolates was carried out through inoculating large volumes of the actinomycetes into $0.85 \%$ Nacl. McFarland units were used in standardizing the inocula. The inocula were applied into the wells of API strips. The strips were incubated at $30^{\circ} \mathrm{C}$ for up to $7 \mathrm{~d}$ [15].

\subsection{Carbon Source Utilization}

The isolated actinomycetes were tested for their ability to utilize D-Glucose, D-Xylose, L-Arabinose, D-Fructose, D-Galactose, Raffinose, D-Mannitol, sucrose, maltose, lactose and cellulose. The isolates were mixed $(1 \% \mathrm{w} / \mathrm{v})$ with the basal medium followed by incubation at $30^{\circ} \mathrm{C}$ for $7 \mathrm{~d}$.

\subsection{Primary Screening of Actinomycetes for Antagonism to Selected Pathogenic Microorganisms}

The antibacterial activity of pure isolates of actinomycetes was determined using streak plate method [16]. Mueller-Hinton agar for bacterial pathogens and sabouraud dextrose agar (SDA) for fungal and yeast pathogens were prepared. The Mueller-Hinton plates were seeded with bacteria while sabouraud dextrose agar were seeded with fungi test organisms by a single streak at a $90^{\circ}$ angle to actinomycete strains. The following pathogens: Staphylococcus aureus (ATCC 25923), Enterococcus faecalis (ATCC 29212), Escherichia coli (ATCC 25922), Pseudomonas aeruginosa (ATCC 27853), Streptococcus pneumoniae (ATCC 49617), Proteus vulgaris (ATCC 49990), Aspergillus niger (ATCC 1015), Fusarium oxysporum (ATCC 16608) and Ustilago maydis (ATCC 14826) were used as test organisms. The test pathogens were retrieved from the culture collection center of the School of Biological Sciences, University of Nairobi. As positive control, vancomycine $(30 \mu \mathrm{g})$ was used for bacteria and crotimazole ( $1 \%$ topical solution) for fungi in addition to use of plain plates as negative control. Antagonism was measured by determination of the size of the inhibition zone in millimeters following incubation of bacteria at $37^{\circ} \mathrm{C}$ for $24 \mathrm{~h}$ and fungal pathogens at $28^{\circ} \mathrm{C}$ for 5 - 25 days [17].

\subsection{Secondary Screening of Actinomycetes for Antagonism to Selected Pathogenic Microorganisms}

M1 agar was prepared and inoculated with isolated actinomycete cultures by 
spread plate technique and incubated at $27^{\circ} \mathrm{C}$ for $5-10$ days. From well grown colonies, $6 \mathrm{~mm}$ agar disks of actinomycetes cultures were cut out using sterile cork borers. Disks were aseptically transferred to Mueller-Hinton agar plates having Staphylococcus aureus (ATCC 25923), Enterococcus faecalis (ATCC 29212), Escherichia coli (ATCC 25922), Pseudomonas aeruginosa (ATCC 27853), Streptococcus pneumoniae (ATCC 49617) and Proteus vulgaris (ATCC 49990). Other disks were similarly transferred to sabouraud dextrose agar seeded with Aspergillus niger (ATCC 1015), Fusarium oxysporum (ATCC 16608) and Ustilago maydis (ATCC 14826). Vancomycine $(30 \mu \mathrm{g})$ for bacteria and crotimazole ( $1 \%$ topical solution) for fungi were used as positive control while plates without the inocula were used as negative control [18].

The inhibition zones $(\mathrm{mm})$ were measured after incubating the bacteria for 24 - $48 \mathrm{~h}$ for $37^{\circ} \mathrm{C}$ and the fungal pathogens for $5-10$ days at $28^{\circ} \mathrm{C}$. Among the actinomycetes isolates, four potent strains that indicated greater activity against Gram-positive, Gram-negative bacteria and fungi and recorded the largest diameter of clear zone were selected for antimicrobial production studies.

\subsection{Extraction of Crude Antimicrobial Compounds}

The selected antagonistic antimicrobial strains were separately inoculated into $3 \mathrm{~L}$ of $\mathrm{M} 1$ broth, and incubated at $28^{\circ} \mathrm{C}$ in a shaker (Gallenkamp, Model 10X 400) (200 rpm) for seven days. After incubation, the broths were filtered through Millipore filters (Millipore Millex-HV Hydrophilic PVDF 0.45 Zm). The filtrates were separately transferred aseptically into a conical flask and stored at $4^{\circ} \mathrm{C}$ for further assay. To the culture filtrate, equal volume of ethylacetate was added separately and centrifuged at $5000 \mathrm{rpm}$ for $10 \mathrm{~min}$ to extract the antimicrobial compound. The compound obtained from each solvent and antimicrobial strain were tested for activity against the test pathogens by agar well diffusion method [19].

\subsection{Separation of Antimicrobial Metabolites}

Ethyl acetate was used in separately dissolving the selected antimicrobials. The solution was concentrated using a vacuum evaporator (Heidolph Laorota, 4001, Buchi Vacuum Conroller V-805) at $40^{\circ} \mathrm{C}(50 \mathrm{rpm})$ to obtain the crude extracts. The crude antimicrobial extracts were collected and dried overnight in a vacuum oven Scwabach, DIN 40050-IP20, V+240, $\mathrm{Hz} 50 / 60$ at $40^{\circ} \mathrm{C}$.

\subsection{Data Analysis}

The data obtained was analyzed using Statistical package for social sciences (SSPS) version 17.0 software. Means of actinomycetes from geothermal vents of region $\mathrm{A}$ and $\mathrm{D}$ were compared using $\mathrm{t}$-test while comparison of the number of actinomycetes isolated using the three types of media were compared using ANOVA at $P=0.05$. 


\section{Results}

\subsection{Isolation of Actinomycetes}

A total of 156 actinomycetes stains were isolated from the soils of Menengai Crater. In region $A$, the actinomycetes isolates varied from $2 \pm 3 \times 10^{8}$ to $9 \pm 2 \times$ $10^{3}$ in starch casein agar (SC), Luria Bertani agar (M1) $\left(5 \pm 2 \times 10^{8}-14 \pm 3 \times\right.$ $\left.10^{3}\right)$ and starch nitrate $(\mathrm{SN})\left(4 \pm 3 \times 10^{8}-14 \pm 2 \times 10^{3}\right)$ (Table 1$)$. On the other hand, variation of the actinomycetes isolates in region B were SC $\left(15 \pm 2 \times 10^{8}\right.$ $\left.25 \pm 3 \times 10^{3}\right), \mathrm{M} 1\left(20 \pm 3 \times 10^{8}-30 \pm 2 \times 10^{3}\right)$ and SN $\left(14 \pm 2 \times 10^{8}-27 \pm 3 \times\right.$ $\left.10^{3}\right)$. In addition, actinomycetes isolated from region $C$ ranged from $(16 \pm 2 \times$ $\left.10^{8}-24 \pm 2 \times 10^{3}\right)$ in SC, M1 $\left(20 \pm 2 \times 10^{8}-31 \pm 3 \times 10^{3}\right)$ and SN $\left(17 \pm 3 \times 10^{8}-\right.$ $\left.28 \pm 2 \times 10^{3}\right)$. Besides, actinomycetes ranges in region D were SC $\left(5 \pm 2 \times 10^{8}-9\right.$ $\left.\pm 2 \times 10^{3}\right), \mathrm{M} 1\left(7 \pm 2 \times 10^{8}-15 \pm 3 \times 10^{3}\right)$ and SN $\left(4 \pm 3 \times 10^{8}-13 \pm 3 \times 10^{3}\right)$. There was a significant difference in the number of actinomycetes isolated using the three types of media $(\mathrm{F}=3.315 P=0.04218)$. Likewise, the number of actinomycetes isolated from region $\mathrm{A}, \mathrm{B} \mathrm{C}$, and $\mathrm{D}$ varied significantly $(\mathrm{F}=27.50 P=$ $0.000)$.

\subsection{Primary Screening of Antimicrobials from Actinomycetes}

A total of 20 actinomycetes showed antagonism against the test pathogenic microorganisms. The mean zone of inhibition for PAN 4 was $8.91 \pm 2 \mathrm{~mm}$, PAN 9 $(12.91 \pm 3)$, PAN $18(11.73 \pm 3)$, PAN $30(21.36 \pm 2)$, PAN $37(21.27 \pm 2)$, PAN $41(23.27 \pm 3)$, PAN $50(8.91 \pm 2)$, PAN $62(5.18 \pm 1)$, PAN $71(8.73 \pm 2)$, PAN $83(6.09 \pm 1)$, PAN $90(6.82 \pm 1)$, PAN $101(11.00 \pm 3)$, PAN 110 (11.18 \pm 3$)$, PAN 117 (10.91 \pm 3$)$, PAN 126 (5.82 \pm 1), PAN 130 (5.82 \pm 1), PAN $132(11.64 \pm$ 2), PAN $137(6.73 \pm 2)$, PAN $150(11.18 \pm 3)$ and PAN $154(20.55 \pm 3 \mathrm{~mm})$ (Table 2). However, there was no significant difference in the zones of inhibition among the isolates $(\mathrm{F}=1.6957 P=0.0838)$ (Figure 1$)$.

Table 1. Number of actinomycetes isolated from soils of Menengai crater using different types of media.

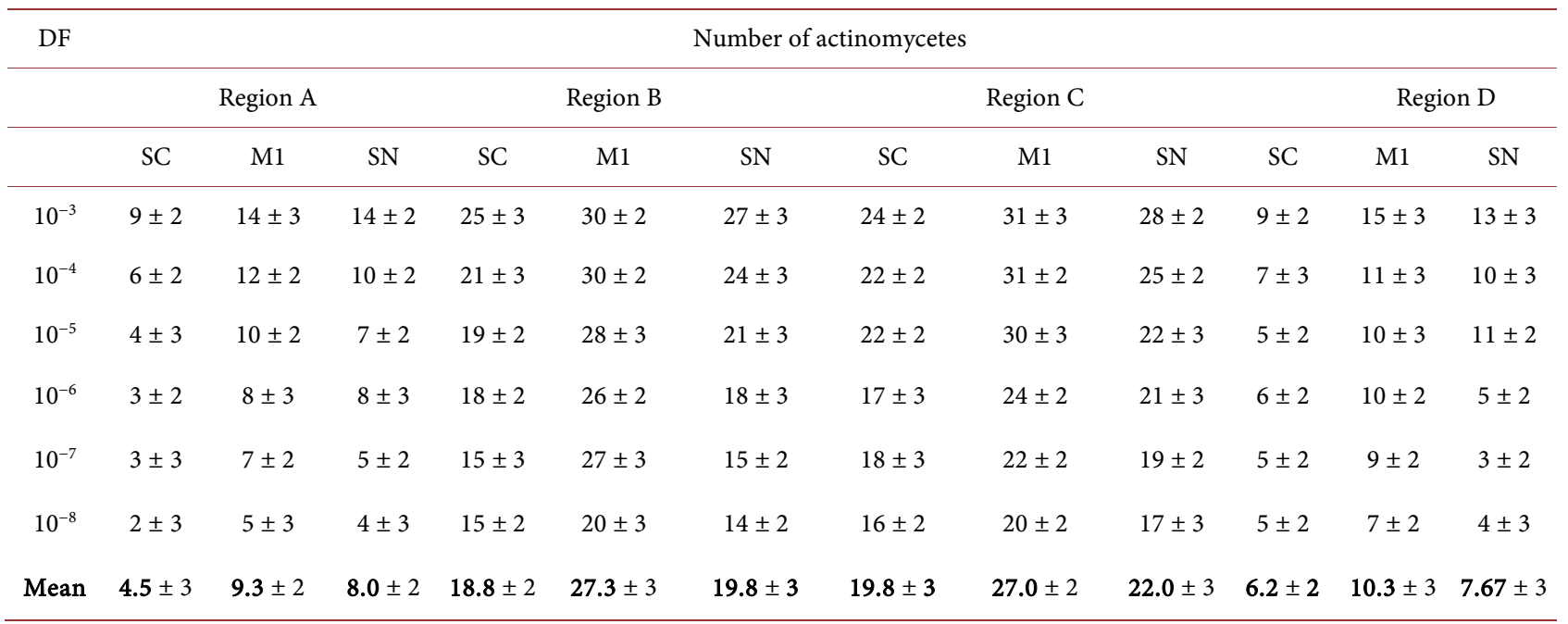

Each value represents the means $( \pm S D)$ of five independent experiments, DF; Dilution factor, M1; Luria Burtani, SN; Starch nitrate. 
Table 2. Zones of inhibition ( $\mathrm{mm}$ ) of selected pathogenic micro-organisms in primary screening of the actinomycetes isolates.

\begin{tabular}{|c|c|c|c|c|c|c|c|c|c|c|c|c|c|}
\hline \multirow{2}{*}{ S. No. } & \multirow{2}{*}{ Isolate } & \multicolumn{11}{|c|}{ Zone of inhibition (mm) } & \multirow[b]{2}{*}{ Mean } \\
\hline & & S. aur & B. sub & E. fae & E. col & K. pne & S. typ & X. cam & E. car & C. $a l b$ & A. Alt & F. oxy & \\
\hline 1 & PAN 4 & $11 \pm 2$ & $15 \pm 3$ & $19 \pm 2$ & $15 \pm 2$ & $0 \pm 0$ & $14 \pm 3$ & $13 \pm 3$ & $10 \pm 2$ & $0 \pm 0$ & $0 \pm 0$ & $0 \pm 0$ & $8.91 \pm 2$ \\
\hline 2 & PAN 9 & $15 \pm 3$ & $14 \pm 3$ & $17 \pm 2$ & $17 \pm 3$ & $10 \pm 2$ & $15 \pm 3$ & $11 \pm 2$ & $10 \pm 2$ & $12 \pm 3$ & $10 \pm 3$ & $11 \pm 3$ & $12.91 \pm 3$ \\
\hline 3 & PAN 18 & $11 \pm 3$ & $17 \pm 2$ & $10 \pm 3$ & $12 \pm 3$ & $9 \pm 2$ & $12 \pm 2$ & $15 \pm 3$ & $11 \pm 3$ & $11 \pm 2$ & $10 \pm 3$ & $11 \pm 2$ & $11.73 \pm 3$ \\
\hline 4 & PAN 30 & $20 \pm 2$ & $23 \pm 3$ & $23 \pm 2$ & $25 \pm 2$ & $19 \pm 3$ & $21 \pm 2$ & $21 \pm 2$ & $21 \pm 2$ & $21 \pm 3$ & $20 \pm 2$ & $21 \pm 2$ & $21.36 \pm 2$ \\
\hline 5 & PAN 37 & $21 \pm 2$ & $20 \pm 2$ & $21 \pm 2$ & $24 \pm 2$ & $21 \pm 2$ & $22 \pm 2$ & $20 \pm 3$ & $22 \pm 3$ & $22 \pm 2$ & $21 \pm 3$ & $20 \pm 3$ & $21.27 \pm 2$ \\
\hline 6 & PAN 41 & $21 \pm 3$ & $25 \pm 3$ & $24 \pm 3$ & $25 \pm 2$ & $23 \pm 3$ & $24 \pm 3$ & $22 \pm 2$ & $24 \pm 2$ & $23 \pm 3$ & $22 \pm 2$ & $23 \pm 2$ & $23.27 \pm 3$ \\
\hline 7 & PAN 50 & $18 \pm 2$ & $0 \pm 0$ & $14 \pm 3$ & $15 \pm 3$ & $15 \pm 3$ & $0 \pm 0$ & $0 \pm 0$ & $0 \pm 0$ & $15 \pm 2$ & $10 \pm 3$ & $11 \pm 3$ & $8.91 \pm 2$ \\
\hline 8 & PAN 62 & $0 \pm 0$ & $0 \pm 0$ & $0 \pm 0$ & $14 \pm 2$ & $0 \pm 0$ & $12 \pm 2$ & $0 \pm 0$ & $0 \pm 0$ & $11 \pm 2$ & $9 \pm 2$ & $11 \pm 2$ & $5.18 \pm 1$ \\
\hline 9 & PAN 71 & $13 \pm 2$ & $13 \pm 3$ & $13 \pm 2$ & $15 \pm 2$ & $0 \pm 0$ & $13 \pm 2$ & $0 \pm 0$ & $0 \pm 0$ & $10 \pm 3$ & $9 \pm 2$ & $10 \pm 3$ & $8.73 \pm 2$ \\
\hline 10 & PAN 83 & $10 \pm 3$ & $15 \pm 2$ & $12 \pm 3$ & $0 \pm 0$ & $0 \pm 0$ & $0 \pm 0$ & $0 \pm 0$ & $0 \pm 0$ & $10 \pm 3$ & $10 \pm 2$ & $10 \pm 2$ & $6.09 \pm 1$ \\
\hline 11 & PAN 90 & $15 \pm 2$ & $16 \pm 3$ & $11 \pm 2$ & $0 \pm 0$ & $0 \pm 0$ & $0 \pm 0$ & $0 \pm 0$ & $0 \pm 0$ & $11 \pm 2$ & $10 \pm 3$ & $12 \pm 3$ & $6.82 \pm 1$ \\
\hline 12 & PAN 101 & $13 \pm 3$ & $12 \pm 2$ & $10 \pm 2$ & $10 \pm 3$ & $12 \pm 2$ & $11 \pm 2$ & $9 \pm 3$ & $12 \pm 3$ & $11 \pm 3$ & $11 \pm 3$ & $10 \pm 3$ & $11.00 \pm 3$ \\
\hline 13 & PAN 110 & $13 \pm 2$ & $14 \pm 3$ & $11 \pm 2$ & $11 \pm 3$ & $10 \pm 3$ & $12 \pm 3$ & $10 \pm 2$ & $10 \pm 3$ & $10 \pm 2$ & $10 \pm 3$ & $12 \pm 2$ & $11.18 \pm 3$ \\
\hline 14 & PAN 117 & $12 \pm 3$ & $11 \pm 2$ & $10 \pm 3$ & $12 \pm 3$ & $13 \pm 3$ & $10 \pm 2$ & $11 \pm 2$ & $11 \pm 2$ & $9 \pm 3$ & $11 \pm 2$ & $10 \pm 2$ & $10.91 \pm 3$ \\
\hline 15 & PAN 126 & $11 \pm 2$ & $11 \pm 2$ & $9 \pm 2$ & $0 \pm 0$ & $0 \pm 0$ & $0 \pm 0$ & $0 \pm 0$ & $0 \pm 0$ & $10 \pm 2$ & $11 \pm 2$ & $12 \pm 3$ & $5.82 \pm 1$ \\
\hline 16 & PAN 130 & $9 \pm 2$ & $9 \pm 3$ & $10 \pm 3$ & $0 \pm 0$ & $0 \pm 0$ & $0 \pm 0$ & $0 \pm 0$ & $0 \pm 0$ & $10 \pm 2$ & $12 \pm 3$ & $14 \pm 2$ & $5.82 \pm 1$ \\
\hline 17 & PAN 132 & $12 \pm 3$ & $11 \pm 2$ & $11 \pm 2$ & $10 \pm 2$ & $10 \pm 2$ & $13 \pm 2$ & $14 \pm 2$ & $14 \pm 2$ & $11 \pm 2$ & $11 \pm 2$ & $11 \pm 2$ & $11.64 \pm 2$ \\
\hline 18 & PAN 137 & $14 \pm 3$ & $11 \pm 3$ & $12 \pm 2$ & $0 \pm 0$ & $0 \pm 0$ & $0 \pm 0$ & $0 \pm 0$ & $0 \pm 0$ & $11 \pm 3$ & $12 \pm 3$ & $14 \pm 3$ & $6.73 \pm 2$ \\
\hline 19 & PAN 150 & $12 \pm 2$ & $10 \pm 3$ & $14 \pm 3$ & $11 \pm 3$ & $12 \pm 3$ & $10 \pm 2$ & $11 \pm 3$ & $10 \pm 3$ & $10 \pm 3$ & $10 \pm 2$ & $13 \pm 3$ & $11.18 \pm 3$ \\
\hline 20 & PAN 154 & $19 \pm 3$ & $20 \pm 2$ & $22 \pm 3$ & $20 \pm 2$ & $21 \pm 3$ & $22 \pm 3$ & $20 \pm 2$ & $19 \pm 2$ & $20 \pm 2$ & $20 \pm 3$ & $23 \pm 2$ & $20.55 \pm 3$ \\
\hline
\end{tabular}

Each value represents the means $( \pm \mathrm{SD})$ of five independent experiments, $S$. aur, Staphylococcus aureus, B. sub; Bacillus subtilis, E. fae, Escherichia faecalis, E. col; Escherichia coli, K. pne, Klebsiella pneuminiae, S. typ; Salmonella typhi, X. cam; Xanthomonas campestris, E. car, Erwinia carotovora, C. alb; Candida albicans, A. alt, Alternaria alternata, F. oxy, Fusarium oxysporum.

\subsection{Secondary Screening for Antimicrobials from Actinomycetes}

There was significant difference in the zones of inhibition among the isolates ( $\mathrm{F}$ $=2.4473 P=0.0089)$ (Table 3$)$. The mean zone of inhibition for PAN 4 was 9.91 $\pm 2 \mathrm{~mm}$, PAN $9(14.18 \pm 3)$, PAN $18(13.18 \pm 3)$, PAN $30(22.27 \pm 2)$, PAN 37 (21.00 \pm 2$)$, PAN $41(24.36 \pm 3)$, PAN 50 (9.82 \pm 2$)$, PAN 62 (5.91 \pm 1$)$, PAN 71 (10.00 \pm 2$)$, PAN $83(6.91 \pm 2)$, PAN 90 (7.82 \pm 1$)$, PAN $101(12.09 \pm 3)$, PAN $110(12.73 \pm 3)$, PAN 117 (11.82 \pm 3$)$, PAN 126 (6.36 \pm 1$)$, PAN 130 (6.45 \pm 1$)$, PAN $132(12.73 \pm 2)$, PAN 137 (6.82 \pm 2$)$, PAN $150(12.72 \pm 2)$ and PAN 154 $(20.91 \pm 3 \mathrm{~mm})$.

\subsection{Screening for Antibiotics of Ethyl Acetate Extracts}

There was a significant difference in the zones of inhibition between the extracts (F $=6.6046 P=0.001338)$. The mean zone of inhibition for PAN 41 was $37.64 \pm$ $2 \mathrm{~mm}$ which was bigger than that of PAN 30 (35.36 \pm 2$)$, PAN $37(34.09 \pm 2)$ and 

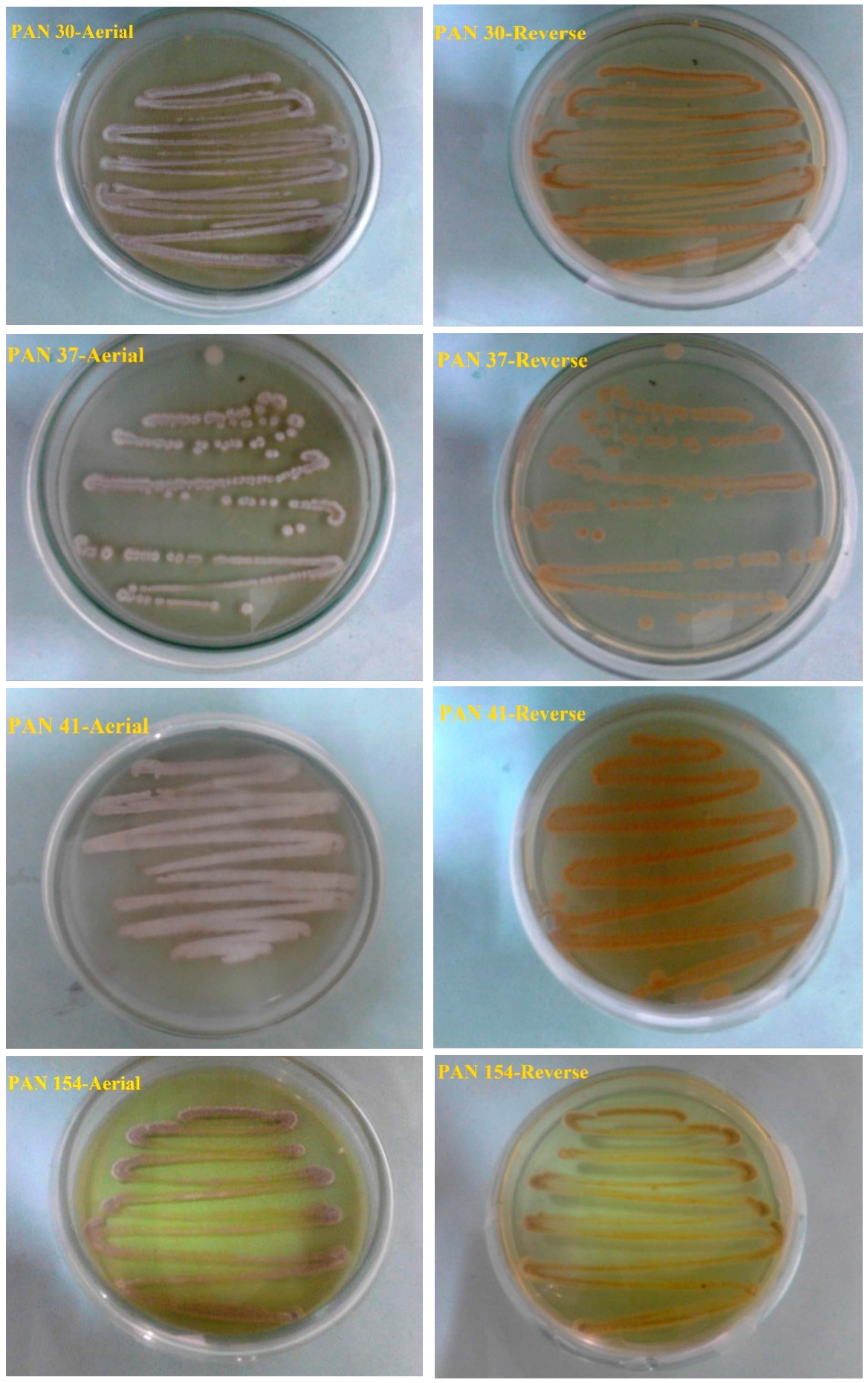

Figure 1. The selected actinomycetes on Luria Bertani agar (M1).

$154(26.27 \pm 2 \mathrm{~mm})$ (Table 4). However, the biggest zone of inhibition was shown by PAN $41(49 \pm 3 \mathrm{~mm})$ against Escherichia coli while the least was $20 \pm$ $3 \mathrm{~mm}$ exhibited by PAN 154 against Bacillus subtilis.

\subsection{Biochemical Test of the Selected Actinomycetes}

All the actinomycetes isolates tested positive for Gram stain, catalase, oxidase, 
Table 3. Zones of inhibition $(\mathrm{mm})$ of the selected pathogenic microorganisms in secondary screening of the actinomycetes isolates.

\begin{tabular}{|c|c|c|c|c|c|c|c|c|c|c|c|c|c|}
\hline \multirow{2}{*}{ S. No. } & \multirow{2}{*}{ Isolate } & \multicolumn{11}{|c|}{ Zone of inhibition (mm) } & \multirow[b]{2}{*}{ Mean } \\
\hline & & S. aur & B. sub & E. fae & E. col & K. pne & S. typ & X. cam & E. car & C. $a l b$ & A. Alt & F. oxy & \\
\hline 1 & PAN 4 & $12 \pm 2$ & $16 \pm 3$ & $22 \pm 2$ & $16 \pm 2$ & $0 \pm 0$ & $16 \pm 3$ & $15 \pm 3$ & $12 \pm 2$ & $0 \pm 0$ & $0 \pm 0$ & $0 \pm 0$ & $9.91 \pm 2$ \\
\hline 2 & PAN 9 & $17 \pm 3$ & $15 \pm 3$ & $19 \pm 2$ & $18 \pm 3$ & $11 \pm 2$ & $16 \pm 3$ & $12 \pm 2$ & $13 \pm 2$ & $14 \pm 3$ & $11 \pm 3$ & $10 \pm 3$ & $14.18 \pm 3$ \\
\hline 3 & PAN 18 & $14 \pm 3$ & $19 \pm 2$ & $11 \pm 3$ & $13 \pm 3$ & $10 \pm 2$ & $13 \pm 2$ & $16 \pm 3$ & $12 \pm 3$ & $13 \pm 2$ & $11 \pm 3$ & $13 \pm 2$ & $13.18 \pm 3$ \\
\hline 4 & PAN 30 & $22 \pm 2$ & $25 \pm 3$ & $24 \pm 2$ & $26 \pm 2$ & $18 \pm 3$ & $20 \pm 2$ & $22 \pm 2$ & $20 \pm 2$ & $25 \pm 3$ & $21 \pm 2$ & $22 \pm 2$ & $22.27 \pm 2$ \\
\hline 5 & PAN 37 & $21 \pm 2$ & $20 \pm 2$ & $22 \pm 2$ & $23 \pm 2$ & $20 \pm 2$ & $21 \pm 2$ & $18 \pm 3$ & $23 \pm 3$ & $22 \pm 2$ & $20 \pm 3$ & $21 \pm 3$ & $21.00 \pm 2$ \\
\hline 6 & PAN 41 & $24 \pm 3$ & $28 \pm 3$ & $25 \pm 3$ & $27 \pm 2$ & $24 \pm 3$ & $23 \pm 3$ & $20 \pm 2$ & $25 \pm 2$ & $25 \pm 3$ & $23 \pm 2$ & $24 \pm 2$ & $24.36 \pm 3$ \\
\hline 7 & PAN 50 & $19 \pm 2$ & $0 \pm 0$ & $15 \pm 3$ & $17 \pm 3$ & $16 \pm 3$ & $0 \pm 0$ & $0 \pm 0$ & $0 \pm 0$ & $18 \pm 2$ & $11 \pm 3$ & $12 \pm 3$ & $9.82 \pm 2$ \\
\hline 8 & PAN 62 & $0 \pm 0$ & $0 \pm 0$ & $0 \pm 0$ & $15 \pm 2$ & $0 \pm 0$ & $15 \pm 2$ & $0 \pm 0$ & $0 \pm 0$ & $13 \pm 2$ & $10 \pm 2$ & $12 \pm 2$ & $5.91 \pm 1$ \\
\hline 9 & PAN 71 & $15 \pm 2$ & $14 \pm 3$ & $15 \pm 2$ & $17 \pm 2$ & $0 \pm 0$ & $16 \pm 2$ & $0 \pm 0$ & $0 \pm 0$ & $12 \pm 3$ & $10 \pm 2$ & $11 \pm 3$ & $10.00 \pm 2$ \\
\hline 10 & PAN 83 & $12 \pm 3$ & $15 \pm 2$ & $16 \pm 3$ & $0 \pm 0$ & $0 \pm 0$ & $0 \pm 0$ & $0 \pm 0$ & $0 \pm 0$ & $10 \pm 3$ & $12 \pm 2$ & $11 \pm 2$ & $6.91 \pm 2$ \\
\hline 11 & PAN 90 & $17 \pm 2$ & $18 \pm 3$ & $14 \pm 2$ & $0 \pm 0$ & $0 \pm 0$ & $0 \pm 0$ & $0 \pm 0$ & $0 \pm 0$ & $12 \pm 2$ & $11 \pm 3$ & $14 \pm 3$ & $7.82 \pm 1$ \\
\hline 12 & PAN 101 & $15 \pm 3$ & $13 \pm 2$ & $11 \pm 2$ & $10 \pm 3$ & $13 \pm 2$ & $11 \pm 2$ & $10 \pm 3$ & $13 \pm 3$ & $14 \pm 3$ & $11 \pm 3$ & $12 \pm 3$ & $12.09 \pm 3$ \\
\hline 13 & PAN 110 & $14 \pm 2$ & $16 \pm 3$ & $13 \pm 2$ & $12 \pm 3$ & $11 \pm 3$ & $13 \pm 3$ & $11 \pm 2$ & $12 \pm 3$ & $13 \pm 2$ & $11 \pm 3$ & $14 \pm 2$ & $12.73 \pm 3$ \\
\hline 14 & PAN 117 & $12 \pm 3$ & $11 \pm 2$ & $12 \pm 3$ & $14 \pm 3$ & $15 \pm 3$ & $12 \pm 2$ & $11 \pm 2$ & $10 \pm 2$ & $11 \pm 3$ & $10 \pm 2$ & $12 \pm 2$ & $11.82 \pm 3$ \\
\hline 15 & PAN 126 & $12 \pm 2$ & $13 \pm 2$ & $10 \pm 2$ & $0 \pm 0$ & $0 \pm 0$ & $0 \pm 0$ & $0 \pm 0$ & $0 \pm 0$ & $10 \pm 2$ & $12 \pm 2$ & $13 \pm 3$ & $6.36 \pm 1$ \\
\hline 16 & PAN 130 & $11 \pm 2$ & $10 \pm 3$ & $10 \pm 3$ & $0 \pm 0$ & $0 \pm 0$ & $0 \pm 0$ & $0 \pm 0$ & $0 \pm 0$ & $11 \pm 2$ & $14 \pm 3$ & $15 \pm 2$ & $6.45 \pm 1$ \\
\hline 17 & PAN 132 & $14 \pm 3$ & $13 \pm 2$ & $12 \pm 2$ & $10 \pm 2$ & $12 \pm 3$ & $14 \pm 2$ & $13 \pm 2$ & $15 \pm 2$ & $12 \pm 2$ & $13 \pm 2$ & $12 \pm 2$ & $12.73 \pm 2$ \\
\hline 18 & PAN 137 & $15 \pm 3$ & $12 \pm 3$ & $13 \pm 2$ & $0 \pm 0$ & $0 \pm 0$ & $0 \pm 0$ & $0 \pm 0$ & $0 \pm 0$ & $11 \pm 3$ & $11 \pm 3$ & $13 \pm 3$ & $6.82 \pm 2$ \\
\hline 19 & PAN 150 & $12 \pm 2$ & $12 \pm 3$ & $15 \pm 3$ & $11 \pm 3$ & $14 \pm 3$ & $11 \pm 2$ & $13 \pm 3$ & $11 \pm 3$ & $14 \pm 3$ & $12 \pm 2$ & $15 \pm 3$ & $12.72 \pm 2$ \\
\hline 20 & PAN 154 & $21 \pm 3$ & $20 \pm 2$ & $21 \pm 3$ & $19 \pm 2$ & $20 \pm 3$ & $21 \pm 3$ & $20 \pm 2$ & $19 \pm 2$ & $23 \pm 2$ & $22 \pm 3$ & $24 \pm 2$ & $20.91 \pm 3$ \\
\hline
\end{tabular}

Each value represents the means $( \pm \mathrm{SD}$ ) of five independent experiments, S. aur, Staphylococcus aureus, B. sub; Bacillus subtilis, E. fae, Escherichia faecalis, E. col; Escherichia coli, K. pne, Klebsiella pneuminiae, S. typ; Salmonella typhi, X. cam; Xanthomonas campestris, E. car, Erwinia carotovora, C. alb; Candida albicans, A. alt, Alternaria alternata, F. oxy, Fusarium oxysporum.

Table 4. Zones of inhibition ( $\mathrm{mm}$ ) of selected pathogenic microorganism to ethyl acetate extracts from the selected actinomycetes.

\begin{tabular}{ccccccccccccc}
\hline \multirow{2}{*}{ Extracts } & \multicolumn{1}{c}{ Zone of inhibition (mm) } & \multicolumn{1}{c}{ Mean } \\
\cline { 2 - 14 } & S. aur & B. sub & E. fae & E. col & K. pne & S. typ & X. cam & E. car & C. alb & A. Alt & F. oxy & \\
\hline PAN 30 & $35 \pm 2$ & $42 \pm 1$ & $41 \pm 3$ & $47 \pm 3$ & $28 \pm 2$ & $37 \pm 3$ & $28 \pm 2$ & $40 \pm 1$ & $30 \pm 3$ & $29 \pm 1$ & $32 \pm 2$ & $35.36 \pm 2$ \\
PAN 37 & $32 \pm 1$ & $41 \pm 2$ & $38 \pm 2$ & $47 \pm 1$ & $25 \pm 2$ & $36 \pm 3$ & $32 \pm 3$ & $41 \pm 2$ & $30 \pm 1$ & $27 \pm 2$ & $26 \pm 3$ & $34.09 \pm 2$ \\
PAN 41 & $39 \pm 1$ & $40 \pm 1$ & $45 \pm 3$ & $49 \pm 3$ & $35 \pm 2$ & $43 \pm 3$ & $38 \pm 2$ & $37 \pm 3$ & $25 \pm 2$ & $30 \pm 3$ & $33 \pm 1$ & $37.64 \pm 2$ \\
PAN 154 & $26 \pm 2$ & $20 \pm 3$ & $25 \pm 2$ & $24 \pm 2$ & $25 \pm 3$ & $28 \pm 2$ & $25 \pm 3$ & $27 \pm 2$ & $28 \pm 2$ & $31 \pm 2$ & $30 \pm 1$ & $26.27 \pm 2$ \\
\hline
\end{tabular}

Each value represents the means $( \pm \mathrm{SD})$ of five independent experiments, S. aur, Staphylococcus aureus, B. sub; Bacillus subtilis, E. fae, Escherichia faecalis, E. col; Escherichia coli, K. pne, Klebsiella pneuminiae, S. typ; Salmonella typhi, X. cam; Xanthomonas campestris, E. car, Erwinia carotovora, C. alb; Candida albicans, A. alt, Alternaria alternata, F. oxy, Fusarium oxysporum. 
urea hydrolysis and gelatin liquefication (Table 5). They were all negative for deaminase, Indole production, beta-galactosidase and lysine decarboxylase. Isolates PAN 30 and 154 were positive for ornithine decarboxylase and citrate utilization while PAN 37 and 41 were negative. In addition, PAN 37, PAN 41 and PAN 154 were positive for hydrogen sulphide production while PAN 30 was negative.

\subsection{Carbon Source Utilization of Actinomycetes Isolated from Menengai Crater}

All the isolates utilized D-glucose and D-galactose. Isolates PAN 30 and Pan 154 utilized sucrose, lactose and maltose while PAN 37 and 41 did not. PAN 154 utilized meso-inositol while PAN30, 37 and 41 were unable to (Table 6). In addition, PAN 30, 37 and 41 utilized L-arabinose while PAN 154 did not. None of the isolates utilized cellulose, D-Mannitol, salicin and raffinose.

\section{Discussion}

Three isolation media-starch casein, Luria Bertani (M1) and starch nitrate agar were used in the isolation of actinomycetes from the soils of Menengai crater. Based on the number of actinomycetes isolates, M1 agar was the best medium for isolation of actinomycetes from this region. This is contrary to studies carried elsewhere [20] [21] [22] [23] which cite starch casein agar as the best isolation medium. This difference can be attributed to differences in the soil sampling region. Menengai crater is a hostile environment implying the possibility

Table 5. Biochemical characteristics of the selected actinomycetes.

\begin{tabular}{|c|c|c|c|c|c|c|c|c|c|c|c|c|}
\hline \multirow[t]{2}{*}{ Isolate } & \multicolumn{12}{|c|}{ Biochemical test } \\
\hline & GS & ONPG & CAT & GLU & LDC & ODC & CIT & $\mathrm{H}_{2} \mathrm{~S}$ & URE & TDA & IND & GL \\
\hline PAN 30 & + & - & + & + & - & + & + & - & + & - & - & + \\
\hline PAN 37 & + & - & + & + & - & - & - & + & + & - & - & + \\
\hline PAN 41 & + & - & + & + & - & - & - & + & + & - & - & + \\
\hline PAN 154 & + & - & + & + & - & + & + & + & + & - & - & + \\
\hline
\end{tabular}

GS: Gram stain, ONPG: beta-galactosidase, CAT: catalase test, GLU: oxidase, LDC: lysine decarboxylase, ODC: ornithine decarboxylase, CIT: citrate utilization, $\mathrm{H}_{2} \mathrm{~S}$ : Hydrogen sulphide production, URE: urea hydrolysis, TDA: deaminase, IND: Indole production, GL: Gelatin liquefication.

Table 6. Carbon source utilization of the selected actinomycetes.

\begin{tabular}{|c|c|c|c|c|c|c|c|c|c|c|c|c|}
\hline \multirow[t]{2}{*}{ Isolate } & \multicolumn{12}{|c|}{ Carbon source } \\
\hline & DG & DGal & Cellulose & $\mathrm{Su}$ & $\mathrm{DM}$ & Lactose & Mal & Salicin & DF & Raffinose & Meso & LA \\
\hline PAN 37 & + & + & - & - & - & - & - & - & + & - & - & + \\
\hline PAN 41 & + & + & - & - & - & - & - & - & + & - & - & + \\
\hline PAN 154 & + & + & - & + & - & + & + & - & + & - & + & - \\
\hline
\end{tabular}

DG; D-Glucose, DGal; D-Galactose, Su; sucrose, DM; D-Mannitol, Mal; Maltose, DF; D-Fructose, Meso; Meso-Inositol, LA; L-Arabinose. 
of variation in the population composition of actinomycetes and therefore need for different nutrients from those isolated from other regions. [24] asserts that the soil nutrient composition leads to differences in nutrient requirements in actinomycetes.

Primary screening is an important procedure for preliminary identification of actinomycetes with antagonistic properties to pathogens. Although many actinomycetes may be isolated from a given soil sample, not all of them may show antagonism to pathogenic microorganism [25]. [26] isolated actinomycetes from the soils of Punjab and obtained a total of 15 isolates out of which 12 indicated antagonism against the tested organisms. This disagreed with the current study. Differences in antimicrobials produced could be a contributing factor to the differences.

In a similar study carried out by [27] in North Iran, the zones of inhibition obtained after carrying out secondary screening were Bacillus subtilis $(8 \mathrm{~mm})$, Staphylococcus aureus $(12 \mathrm{~mm})$, E. coli $(9 \mathrm{~mm})$ and Klebsiella spp. (0.00). These were lower than the ones obtained in the current study. This could be attributed to differences in the genetic codes that are responsible for production of antimicrobials. [28] explains that the genetic constitution of a particular actinomycete to a large extent dictates the antimicrobials produced.

This study obtained bigger zones of inhibition after testing for sensitivity of the pathogens to ethyl acetate extracts than previous studies carried out in other regions [29]. According to [30], different actinomycetes yield different types of antimicrobials Further, [31] asserts that the environment in which actinomycetes are growing in to a great extent, influences the metabolic activities of the organisms thus influencing the types of antimicrobials produced.

Results on biochemical tests obtained in this study slightly differ with those of previous studies carried out elsewhere [32] [33] [34]. [35] explains that different stains of actinomycetes present varying biochemical reactions. However, the results of carbon utilization obtained in this study agreed with a previous study carried out in a foot-hill of Western Ghats in India [36]. This may have been caused by similarity in carbon source requirement as explained by [37].

\section{Conclusion}

Actinomycetes were successfully isolated from Menengai crater. Although a total of 156 actinomycetes were isolated, 20 actinomycetes showed antagonism against the test pathogens. Four antimicrobial metabolites showed the highest antagonism against the test pathogens. The study indicated that Menengai crater has the potential of producing actinomycetes that can produce antimicrobials with high capability of treating diseases caused by tested pathogenic microorganisms.

\section{Recommendation}

There is need to purify and characterize the antimicrobials obtained from the 
present study.

\section{Conflict of Interest}

The authors declare no conflict of interest

\section{References}

[1] Amit, P. and Sunil, K. (2015) Antibiotic Activity of Antimicrobial Metabolites Produced from Soil Microorganisms: An Overview. International Journal of Pharmaceutical Research and Allied Sciences, 4, 28-32.

[2] Sudha, S.K.S. and Hemalatha, R. (2015) Isolation and Screening of Antibiotic Producing Actinomycetes from Garden Soil of Sathyabama University, Chennai. Asian Journal of Pharmaceutical and Clinical Research, 8, 110-114.

[3] Houssam, M.A. (2015) Biochemical Studies on Antibiotic Production from Streptomyces sp.: Taxonomy, Fermentation, Isolation and Biological Properties. Journal of Saudi Chemical Society, 19, 12-22. https://doi.org/10.1016/j.jscs.2011.12.011

[4] Mobeen, S., Girija, S.G., Iswarya, M. and Rajitha. P. (2017) Isolation and Characterization of Bioactive Metabolites Producing Marine Streptomyces parvulus Strain Sankarensis-A10. Journal of Genetic Engineering and Biotechnology, 15, 87-94. http://doi.org/10.1016/j.jgeb.2017.02.004

[5] Shalini, R.V. and Amutha, K. (2015) Isolation and characterization of antifungalactinomycete from Thiruporur forests. I International Journal of Pharma and Bio Sciences, 6, 750-758. https://doi.org/10.5897/AJARX11.071

[6] Winanda, H., Arinthip, T., Wasu, P. and Kannika, D. (2016) Actinomycetes from Eucalyptus and Their Biological Activities for Controlling Eucalyptus Leaf and Shoot Blight. Microbiological Research, 188, 42-52. http://doi.org/10.1016/j.micres.2016.04.011

[7] Omar, M., Mourad, B. and Ibrahim, A. (2015) Identification and Preliminary Characterization of Non-Polyene Antibiotics Secreted by New Strain of Actinomycete Isolated from Sebkha of Kenadsa, Algeria. Asian Pacific Journal of Tropical Biomedicine, 5, 438-443.https://doi.org/10.1016/j.apjtb.2015.04.002

[8] Rajeswari, P., Jose, P.A., Amiya, R. and Jebakumar, S.R.D. (2015) Characterization of Saltern Based Streptomyces sp. and Statistical Media Optimization for Its Improved Antibacterial Activity. Frontiers in Microbiology, 2014, 00753. https://doi.org/10.3389/fmicb.2014.00753

[9] Deshmukh, A.A. and Vidhale, N.N. (2014) Screening of Antimicrobial Actinomycetes from Saline Belt of Vidarbha Region. International Journal of Life Sciences, 2, 355-358.

[10] Udaya, P., Rao, C.S. and Satish, B.R. (2013) Studies on L-Asparaginase Production by Using Staphylococcus capitis. Journal of Chemical, Biological and Physical Sciences, 3, 201-209.

[11] Janaki, T., Nayak, B.K. and Ganesan, T. (2014) Different Pre-Treatment Methods in Selective Isolation of Actinomycetes from Mangrove Sediments of Ariyankuppam Back Water Estuary, Puducherry. International Journal of Advanced Research in Biological Sciences, 1, 154-163.

[12] Pooja, S., Rajesh, K., Mahesh, S.Y., Nityanand, M. and Dilip, K.A. (2015) Isolation and Characterization of Streptomyceteswith Plant Growth Promoting Potential from Mangrove Ecosystem. Polish Journal of Microbiology, 64, 339-349.

https://doi.org/10.5604/17331331.1185232 
[13] Waithaka, N.P., Maingi, J.M. and Nyamache, A.K. (2015) Physico-Chemical Analysis, Microbial Isolation, Sensitivity Test of Theisolates and Solar Disinfection of Water Running in Community Taps and River Kandutura in Nakuru North Sub-County, Kenya. The Open Microbiology Journal, 9, 117-124. https://doi.org/10.2174/1874285801509010117

[14] Sahar, Y.I. and Magda, M.A. (2016) Isolation of Anti-Fungal Agent from a Soil Inhabitant Streptomyces albaduncus-M51 and Its Efficacy against Osmophilic Food Spoilage by Saccharomyces cerevisiae. Journal of Environmental and Occupational Science, 5, 38-46. https://doi.org/10.5455/jeos.20160530063639

[15] Juliah, K.A., Markus, G.K., Manfred, R., Peter, S., Hans-Peter, K. and Hamadi, I.B. (2015) Belliellakenyensis sp. nov., Isolated from an Alkaline Lake. International Journal of Systematic and Evolutionary Microbiology, 65, 457-462. https://doi.org/10.1099/ijs.0.066951-0

[16] Sathya, R. and Ushadevi, T. (2014) Industrially Important Enzymes Producing Streptomyces sp. from Mangrove Sediments. International Journal of Pharmacy and Pharmaceutical Sciences, 6, 233-237.

[17] Rita, N.W. and Arun, T.P. (2013) Isolation and Characterization of Bioactive Actinomycetes from Soil in and around Nagpur. International Journal of Pharmaceutical Sciences and Research, 4, 1428-1433.

[18] Ahmed, I.K., Eltahir, H.B. and Humodi, A.S. (2016) Streptomyces: Isolation, Optimization of Culture Conditions and Extraction of Secondary Metabolites. International Current Pharmaceutical Journal, 5, 27-32.

[19] Al-Hulu, S.M. (2013) Study Effects of Some Parameters on Antifungal Activity for Streptomyces spp. Journal of Kerbala University, 11, 254-260.

[20] Attimarad, S.L, Ediga, L.G., Karigar, A.A., Karadi, R., Chandrashekhar, N. and Shivanna, C. (2012) Screening, Isolation and Purification of Antibacterial Agents from Marine Actinomycetes. International Current Pharmaceutical Journal, 1, 394-402. https://doi.org/10.3329/icpj.v1i12.12448

[21] Kumar, P.S., Al-Dhabi, N.A., Duraipandiyan, V., Balachandran, C., Kumar, P.P. and Lgnacimuthu, S. (2014) In Vitro Antimicrobial, Antioxidant and Cytotoxic Properties of Streptomyces lavendulae Strain SCA5. BMC Microbiology, 14, 291-300. https://doi.org/10.1186/s12866-014-0291-6

[22] Dhanam, J.G. and Kannan, S. (2015) Depiction and Screening of L-asparaginase Producing Actinomycetes Isolated from the Soil Samples of Termite Mounts. International Journal of Advanced Scientific and Technical Research, 5, 304-310.

[23] Samy, G.B., Sujitha, S., Thyagarajan, R. and Jegatheesan, K. (2015) Isolation of Streptomyces Species from Soil and Its Medium Optimization for Microbial Transglutaminase Production by Box-Behnken Design. Journal of Ecosystem and Ecography, 6, 175-190.

[24] Sukhvir, K., Harjot, P.K. and Gagandeep, K. (2016) Isolation and Characterization of Antibiotic Producing actinomycetes from Agriculture Soil. World Journal of Pharmaceutical Sciences, 5, 1109-1117.

[25] Akhgari, Z., Heshmatipour, Z. and Mahtabipour, M.R. (2014) Anti-Bacterial Potential of New Streptomyces sp. nov Isolated from Hot-Springs North of Iran. Advances in Environmental Biology, 8, 2008-2011.

[26] Charousová, I., Medo, J., Halenárová, E. and Javoreková, S. (2017) Antimicrobial and Enzymatic Activity of Actinomycetes Isolated from Soils of Coastal Islands. Journal of Advanced Pharmaceutical Technology \& Research, 8, 46-51. 
[27] Saker, R., Noureddine, B., Atika, M., Abdelghani, Z., Peter, S., Cathrin, S., Hans-Peter, K. and Nasserdine, S. (2015) Actinopolysporabiskrensis sp. nov., a Novel Halophilic Actinomycete Isolated from Northern Sahara. Current Microbiology, 70, 423-428. https://doi.org/10.1007/s00284-014-0740-3

[28] Meeta, M. and Ekta, M. (2015) Antimicrobial Activity of Isolates of Actinomycetes from Soils of Semi-Arid Regions of Rajasthan. World Journal of Pharmaceutical Sciences, 4, 1549-1556.

[29] Polpass, A.J., Solomon, R. and David, J. (2013) Non-Streptomycete Actinomycetes Nourish the Current Microbial Antibiotic Drug Discovery. Frontiers in Microbiology, 4, 240.

[30] Ilayaraja, S., Rajkumar, J., Swarnakumar, N.S., Sivakumar, K., Thangaradjou, T. and Kannan, L. (2014) Isolation of Two Thermophilic Actinobacterial Strains Mud Volcano of the Baratang Island, India. African Journal of Microbiology Research, 8, 40-45. https://doi.org/10.5897/AJMR09.126

[31] Wink, J. and Mohammadipanah, F. (2015) Actinobacteria from Arid and Desert Habitats: Diversity and Biological Activity. Frontiers in Microbiology, 6, 541-516.

[32] Yoke-Kqueen, C., Learn-Han, L., Cheng-Yun, C. and Vui-Ling, M.W. (2015) Isolation, Identification and Screening of Actinobacteriain Volcanic Soil of Deception Island (the Antarctic)for Antimicrobial Metabolites. Polish Polar Research, 36, 67-78.

[33] Kothagorla, V.R., Palla, M., Botcha, S. and Tamanam, R.R. (2017) Purification and Structural Elucidation of Three Bioactive Compounds Isolated from Streptomyces coelicoflavus BC 01 and Their Biological Activity. Biotech, 7, 1-12.

[34] Astalakshmi, A., Thangapandian, V. and Lingakumar, K. (2014) Isolation and Characterization of Actinomycetes from the Soil of Devathanam-A Foot-Hill of Western Ghats. International Journal of Pharmaceutical Sciences Review and Research, 3, 15-20.

[35] Garima, A. and Jugmendra, S. (2016) Statistical Analysis of Actinomycetes Isolated from the Haridwar Region of Uttarakhand (India). Journal of Drug Delivery and Therapeutics, 6, 31-36. http://jddonline.info/15.07.2016

[36] Telugu, V., Kalva, M.S. and Charyulu, P.B.B. (2014) Taxonomic Studies and Phylogenetic Characterization of Potential and Pigmented Antibiotic Producing Actinomycetes Isolated from Rhizosphere Soils. International Journal of Pharmacy and Pharmaceutical Sciences, 6, 511-551.

[37] Zahir, H., Hamadi, F., Mallouki, B. and Imziln, H.L. (2016) Effect of Salinity on the Adhesive Power Actinomycetes in Soil. Journal of Materials and Environmental Science, 7, 3327-3333. 